NASA Technical Memorandum 110249

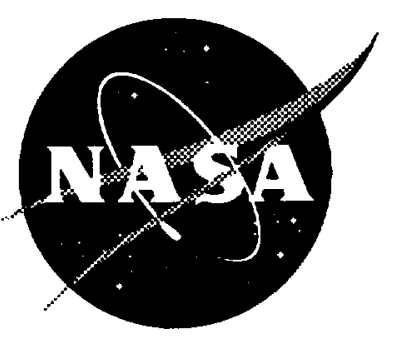

\title{
STS-74/MIR PHOTOGRAMMETRIC APPENDAGE STRUCTURAL DYNAMICS EXPERIMENT
}

Michael G. Gilbert

Sharon S. Welch

Langley Research Center

Hampton, Virginia

\section{April 1996}

National Aeronautics and Space Administration Langley Research Center

Hampton, Virginia 23681-0001 


\title{
STS-74/MIR Photogrammetric Appendage Structural Dynamics Experiment
}

\author{
Michael G. Gilbert ${ }^{1}$ and Sharon S. Welch ${ }^{2}$ \\ NASA Langley Research Center \\ Hampton, VA. 23681-0001
}

\begin{abstract}
The Photogrammetric Appendage Structural Dynamics Experiment (PASDE) is an International Space Station (ISS) Phase-I risk mitigation experiment. Phase-I experiments are performed during docking missions of the U.S. Space Shuttle to the Russian Space Station Mir. The purpose of the experiment is to demonstrate the use of photogrammetric techniques for determination of structural dynamic mode parameters of solar arrays and other spacecraft appendages. Photogrammetric techniques are a low cost alternative to appendage mounted accelerometers for the ISS program. The objective of the first flight of PASDE, on STS-74 in November 1995, was to obtain video images of Mir Kvant-II solar array response to various structural dynamic excitation events. More than 113 minutes of high quality structural response video data was collected during the mission. The PASDE experiment hardware consisted of three instruments each containing two video cameras, two video tape recorders, a modified video signal time inserter, and associated avionics boxes. The instruments were designed, fabricated, and tested at the NASA Langley Research Center in eight months. The flight hardware was integrated into standard Hitchhiker canisters at the NASA Goddard Space Flight Center and then installed into the Space Shuttle cargo bay in locations selected to achieve good video coverage and photogrammetric geometry.

\section{Introduction}

The Photogrammetric Appendage Structural Dynamics Experiment (PASDE)

\footnotetext{
${ }^{1}$ Senior Research Engineer, Structural Dynamics Branch. Senior Member AIAA.

2 Senior Research Engineer, Guidance and Controls Branch. Senior Member AIAA.
}

has been developed to mitigate technical risk and cost associated with on-orbit measurement of solar array and other flexible appendage structural responses for the International Space Station (ISS) program. The experiment objectives are to demonstrate photogrammetric structural response measurement methods for solar arrays which may be articulating, to provide engineering data on solar array designs similar to those expected to be used on the ISS, and to verify that routine on-orbit spacecraft operational events provide sufficient excitation for structural response testing. PASDE flew inspace on the Space Shuttle STS-74 mission in November 1995 to the Russian Space Agency MIR spacecraft, and a follow-on flight is currently manifested for the STS-86 mission to MIR in August 1997.

On-orbit measurements of spacecraft structural response are often desired or necessary for structural verification and loads prediction validation. Typically, acceleration response time-history data is collected via onboard accelerometers, conditioned, and transmitted (downlinked) to the ground for further processing. From the acceleration time history data, structural dynamic characteristics (structural mode frequencies, damping, and mode shapes) are determined using structural identification algorithms such as the Eigensystem Realization Algorithm (ERA) [1].

The use of photogrammetric measurements is a low cost alternative to dedicated accelerometer structural response measurements, especially when measurements are required for articulating or rotating spacecraft components such as solar arrays or thermal radiators. Elimination of accelerometers, power and data wiring, signal conditioning and digital conversion electronics, etc., can greatly simplify the spacecraft electrical design and integration, 
with corresponding reduction in spacecraft cost.

For the International Space Station (ISS), Figure 1, on-orbit structural response measurements are required for loads validation and verification of structural mathematical models. Accelerometer-based measurements of the U.S. primary truss and modules are currently being planned, however, accelerometer measurement of the U.S. solar arrays are not being considered because of cost and resource impacts. Since the current ISS design calls for numerous video cameras mounted at various external points, photogrammetric measurements of solar array structural responses are a potential low-cost alternative for obtaining the necessary data. In addition to providing data on the U.S. solar arrays and thermal radiators, the planned ISS video system should also be capable of making measurements on components provided by ISS international partners.

The International Space Station program is divided into three phases. Phase I, the Risk Mitigation Program phase, involves flights of the U.S. Space Shuttle to the Russian Space Agency Mir space station. Phase II of the ISS program is an assembly phase including the initial on-orbit assembly through to a permanently crewed capability. Phase III of the ISS program is from the initial permanently manned capability through final assembly (assembly complete). During Phase I, the technical risk and cost of technologies, component designs, and operational practices planned for ISS are to be reduced through flight testing and verification. Because photogrammetric structural response measurement techniques have the potential to reduce ISS cost, but had not been demonstrated on-orbit for arrays and other appendages undergoing rigid-body motions relative to the camera system, the PASDE experiment was advocated and selected as a Phase I risk mitigation experiment. The experiment concept was to use six video cameras at three locations in the Shuttle payload bay to record data on $\mathrm{Mir}$ solar array structural response during docked Shuttle/Mir operations, with post-flight data processing to establish measurement feasibility and resolution.

The NASA Langley Research Center (LaRC) was funded by the NASA Headquarters Code Office of Space Access and Technology for hardware development and first flight of PASDE as a Hitchhiker [2] payload. The NASA Goddard Space Flight Center (GSFC) was funded to provide Hitchhiker canisters, windows, and payload services for the PASDE experiment. For the STS-74 mission, PASDE was manifested by the GSFC Hitchhiker Program with the Glo4 experiment as the Hitchhiker Glo-4/PASDE [3] or GPP payload. For the planned flight on STS-86, the seventh mission of Shuttle to Mir, PASDE hardware will be used to obtain measurements in conjunction with the Mir Structural Dynamics Experiment (MiSDE) Risk Mitigation Experiment. Funding for the STS-86 flight of PASDE is provided directly by the ISS Phase I program.

The remainder of this paper is organized as follows. The concept of photogrammetric structural response measurements is discussed first, followed by specific measurement requirements for the PASDE experiment. The design and fabrication of hardware to meet the measurement requirements is covered next, followed by the STS-74 mission science objectives and an overview of STS-74 mission operations. Flight tapes containing the photogrammetric quality video data had not been retrieved at the time this paper was prepared. Data presented herein is limited to on-orbit video data downlinked from the Shuttle during the actual mission.

\section{Photogrammetric Structural Response Measurement}

Photogrammetry is defined as "the science of making reliable measurements by the use of photographs..." [4]. In the case of a single photograph or image, certain precise measurements of an object in the image, such as geometrical size, can be made under appropriate conditions. With several images of an object, again under appropriate conditions, the orientation and/or position of the object with respect to the positions of the 
cameras can be determined using a triangulation process [5]. The precision of the position or orientation determination is dependent on the quality and number of images. At least two independent images are required for triangulation, with more images leading to higher precision. If the object of interest is moving, determination of the position or orientation as a function of time requires a sequence or series of images from each camera location and triangulation at each time of interest. Measurement of timevarying structural response, which is of interest here, is an example of this third type of photogrammetric measurement.

The recent advent of low-cost, charge-coupled-device (CCD) video cameras, video recorder systems, and digital image processing techniques have facilitated photogrammetric measurement of structural dynamic response [e.g. 6,7]. In the 1984 Solar Array Flight Experiment (SAFE) for example, photogrammetric measurements of a solar array cantilevered from the cargo bay of the Space Shuttle were made from video recorded by the Shuttle's on-board camera systems. The position of the array in the cargo bay was fixed with respect to the Shuttle camera system in this experiment. As shown in Figure 2, the motion recorded in the SAFE video images was directly proportional to the structural deformation of the array as it responded to various structural excitation sources.

For the case of the International Space Station solar arrays, there will be relative, rigid-body motion of the solar array with respect to the positions of the photogrammetric cameras. This motion is due to articulation and/or rotation of the array as it tracks the sun in Earth orbit. Thus the motion measured by a photogrammetric system for ISS solar arrays will consist of combined rigid-body and structural deformations, as shown in Figure 3. To extract the structural motion from the combined motion, the apparent "rigid-body" motion component must be determined. This can be accomplished by determining the motion of points on the solar array which can reasonably be expected to have little structural deformation (such as near the root), and using this measurement to predict a "rigidbody" orientation and position of the array from known array geometry. Subtracting the predicted "rigid-body" motion from the total measured motion leaves an estimate for the structural deformation. The PASDE experiment was designed to make relative motion structural measurements, including the consideration of rigid-body motion, as defined in Figure 3.

\section{PASDE Measurement Requirements}

The hardware requirements for onorbit photogrammetric spacecraft structural response measurements are dependent on the size and orientation of the appendage(s) of interest, the lighting conditions, the distances and three dimensional relationship of the imaging devices, and the number of desired mode parameter sets (frequencies, damping, and shapes) desired.

For the PASDE hardware development, a general sense of potential flight opportunities and possible targets and geometry was used to generate a set of design requirements. These potential flight opportunities involved imaging solar arrays on the Russian Mir space station during Space Shuttle docking flights. For these opportunities, photogrammetric distances ranging from 10 to 20 meters ( 30 to 60 feet) were expected, measuring from 2 or 3 Shuttle cargo bay locations. Identification of the first 3 or 4 solar array mode parameter sets was desired, leading to the hardware design requirements listed in Table 1.

From analytical finite element structural models of the coupled (docked) Shuttle/Mir spacecraft, solar array responses to expected on-orbit excitation events were computed. These results in conjunction with solid-body viewing models and likely Shuttle payload bay locations led to the PASDE requirement to resolve $0.25 \mathrm{~cm}(0.1 \mathrm{inch})$ motions at the expected viewing distances. To identify both bending and torsion mode parameters while extracting relative array motions, a minimum of six time history data points are required. Desired ranges of locations for the six points were defined and target regions for pointing the cameras were 
established, as illustrated in Figure 4. In Figure 4, the arrows and dimensions establish the six approximate locations, indicated by small circles, with respect to the length and width of the array. The larger viewing circles indicate the desired viewing areas for the cameras, which encompass the minimum number of points for which time history data is needed. Actual data points for flight data analysis will be determined after the flight based on the obtained images, contrast, identifiable features, etc. It is expected that time history data will be obtained for many more than the minimum number of points.

The other requirements listed in Table 1 were defined qualitatively, accounting for the typical types of data characteristics needed for good identification of structural modes. These included the amount of free decay response following excitation, the time correlation of the video images with each other, and the sampling rate. The minimum 10 Hertz sampling rate provides a 10 to 1 frequency ratio with respect to the highest frequency mode that can reasonably be expected to be identified. Standard video data is 30 Hertz.

\section{Hardware Design}

Based on the length of the Mir solar arrays and the viewing geometry's possible in the Shuttle payload bay, a PASDE design incorporating six video cameras in three Hitchhiker canisters located in the Shuttle payload bay was selected. Two cameras are in each Hitchhiker canister, one aimed and focused at the root of the array, and the other at the tip of the array. It was also decided that the PASDE hardware for each canister would be identical, with adjustable camera mounts to allow for pre-flight pointing. A photo of an assembled flight unit is shown in Figure 5, prior to installation into a Hitchhiker canister.

PASDE uses Pulnix Model TM-9701 monochrome video cameras with CCD array sizes of $764 \times 486$ pixels. Each camera has a Schneider 50 millimeter focal length lens with a motor driven iris to adjust for lighting conditions. Lens focus is adjusted on the ground prior to flight and cannot be changed on-orbit. The camera/lens combination has a \pm 5 degree field-of-view. Associated with each camera is a Teac Model V-80AB-F Hi8 video tape recorder providing two hours of video recording time. A single modified video time code inserter from Sekai, a single Power Conversion and Distribution Unit (PCDU) from Vicor, and a single LaRC designed and built Interface and Control Unit (ICU) are also used for each flight unit. The IRIG-B time inserters convert time code from the Shuttle into a video format and add the result to the video signal prior to recording. This ensures that the six recorded video signals can be time correlated to within a single video frame. The ICU provides the interface between the PASDE hardware and the standard Hitchhiker avionics. It processes 8 different bi-level commands from the Hitchhiker to activate the hardware, to initiate and stop recording, and to establish lens iris positions. Rewinding and/or replaying the data tapes on-orbit using the bilevel command interface is not possible.

Three sets of identical flight hardware were designed, fabricated, assembled, and tested at the NASA Langley Research Center beginning in October of 1994. Each unit was subjected to acceptance level vibration, electro-magnetic interference, thermalvacuum, and functional tests. The units were delivered to GSFC in early June 1995, slightly more than eight months from initiation of design. Integration at GSFC with the actual Hitchhiker canisters followed. Once integrated, the canisters were sealed with a large aperture window for the upper endplate and pressurized with dry nitrogen.

Upon delivery to the NASA Kennedy Space Center in July 1995, a pre-flight photogrammetric calibration of the system was performed using a simulated solar array assembly. At the conclusion of the photogrammetric calibration tests, the simulated solar array assembly was vibrated and data was recorded on the flight hardware as an approximation of the on-orbit experiment. Results from this pre-flight ground experiment are documented in Ref. 8. The final operational test of the PASDE hardware prior to flight occurred after 
installation and electrical connection of the canisters with the Shuttle Atlantis during September 1995.

\section{Experiment Overview}

The structure of interest for the PASDE experiment on the STS-74 mission was the Mir Kvant-II module lower solar array as shown in Figure 6. During the docked portions of the combined Shuttle/Mir mission, this array was outboard of the Shuttle on the port side, slightly ahead of the wing. The three Hitchhiker canisters containing the PASDE flight hardware were mounted aft of the expected Kvant-II array position in Shuttle payload Bay 6 (port side), Bay 7 (starboard side), and Bay 13 (port side). Expected images generated pre-flight from computer graphics models of the docked Shuttle/Mir spacecraft for the Bay 7 root and tip cameras are shown in Figure 7 (for the orientation of the array with respect to Kvant-II shown in Figure 6).

From the Shuttle/Mir mission plans, a set of on-orbit were identified pre-flight as likely to result in detectable structural motion of the imaged solar array. These events included the actual docking of the Shuttle to Mir, a series of Shuttle Primary Reaction Control System (PRCS) jet firings planned for flight control system test purposes, the day/night and night/day terminator crossings, and solar array sun track rotations. The terminator crossings were of interest because of potential thermal responses due to the rapid change from full sunlight to complete darkness and back to full sunlight. The solar array sun track rotations were considered to be a potential source of structural excitation because, unlike many U.S. systems, the Mir arrays track the sun in approximately 15 degree increments at relatively high rotational rates. A total of 100 minutes of a possible 120 minutes of video data recording capability was allocated for the identified events, with the remaining time available for contingency, data collection events-ofopportunity, and post-flight calibration purposes.

Additional sources of structural response data that could be correlated with the PASDE measurements were identified. One source of such data was the normal Shuttle downlink data, including for example PRCS jet status's and Inertial Measurement Unit angular rates and translational velocities. Another source of supporting data was from 22 accelerometers mounted at various locations throughout the Mir modules, however this data could only be obtained during excitation events which occurred over Russian ground stations.

\section{Mission Operations}

PASDE mission operations were conducted during the STS-74 mission from the Hitchhiker Payload Operations Control Center (POCC) at NASA GSFC. The PASDE hardware was normally commanded directly from the POCC by Hitchhiker personnel via a command link through Mission Control at NASA Johnson Space Center and then to the Shuttle via Tracking and Data Relay System (TDRS) satellites. Commands were sent by the Hitchhiker personnel on request of the PASDE operations team. During times when direct commanding of PASDE through the command link was not possible, commands to start and stop recording could be preloaded on the Shuttle to execute at specific times. This capability was used several times during the mission when commanding problems due to antenna blockages or TDRS zone-of-exclusion (ZOE) events were predicted to exist.

Within the POCC, the PASDE operations team had electrical current and temperature information from the flight hardware, and a limited capability to view the video signal from any one of the six cameras via Shuttle downlink. A variety of additional sources of standard real-time mission information, including mission control voice loops, vehicle state and attitude information, graphical displays, etc., were also available.

Table 2 summarizes actual PASDE operations during the STS-74 flight. Events are listed by a Mission-Elapsed-Time (MET) reference in day:hours:minutes format, amount of data recorded in minutes:seconds format, and a short description. Each of the 
major events or blocks of events is described in more detail below, using the same formats for MET and record time. During the mission, tape usage was tracked by individual canister, as canister commanding occurred serially and could thus result in varying tape usage times. However, for the purpose of tracking record time and computing remaining tape, the maximum time used by any one of the three canisters during the event was used.

PASDE Activation - STS-74 was launched at 7:30:43 am, EST on November 11,1995 . The crew of the Shuttle performed the Glo-PASDE Payload activation procedure at MET 0:03:25, providing power to the PASDE hardware from the Hitchhiker avionics system. Once video downlink capability was established with the orbiter, the video signal from each camera was viewed on the ground with a monitor. All six cameras were verified to be operating properly. As the Shuttle was oriented with the payload bay pointed to earth, these initial downlink views were of the Atlantic Ocean and Western Africa. Following verification of camera operation, each of the three instruments was individually commanded to collect data for 1-2 minutes to verify operation of the tape recorders.

Camera Alignment. Contingency Data Collection, and Pre-Docking Lighting Calibration - Prior to docking with Mir, the Shuttle Remote Manipulator System (RMS) robotic arm was to be placed in the fields-ofview of the six cameras to assist in calibrating camera iris settings. Since the RMS typically exhibits visible vibrational motion when maneuvering, the final placement of the RMS into the desired calibration orientation was identified as a source of contingency data, in the event docking with Mir did not occur. The final RMS positioning maneuver was recorded starting at MET 1:04:45 for 11:35. Following the RMS positioning, the video signal from each of the six cameras was downlinked to the ground. Each of the scenes was compared with predicted views developed before flight from the desired RMS orientation as a check that camera alignment (pointing angles) did not change during launch. All six camera views were as predicted prior to flight. Over the next several hours, the Shuttle attitude was adjusted to the attitude expected at docking with Mir and a lighting calibration to finalize lens iris settings for docking was completed.

Docking - The nominal mission plans called for docking of the Shuttle with the Mir spacecraft at MET 2:15:57 over central Russia just before orbital sunset. Immediately prior to docking, a loss of direct payload commanding from the POCC was expected, lasting from approximately 7 minutes prior to docking to 2 minutes prior to docking. Commands to start the PASDE tape recorders were uplinked to the Shuttle computers prior to the command link loss for automatic execution five minutes before docking. The docking proceeded normally, with the PASDE recorders beginning to record on command of the Shuttle at 2:15:52, followed by docking on schedule at 2:15:57.

PASDE data recording continued until 2:15:82 when the recorders were commanded to stop recording directly from the POCC. It was not possible to view PASDE video data during or after the docking, however video data of the docking from several Shuttle payload bay cameras was downlinked and clearly showed dynamic motion of the KvantII solar array.

\section{PRCS Tests and PRCS Attitude} Maneuver - A series of primary reaction control jet firing tests for Shuttle attitude control purposes were conducted with the Shuttle docked to Mir. These jet firing tests were predicted to provide structural excitation of the Kvant-II solar array. The first of these tests occurred shortly after docking, consisting of four sequences of single or dual jet firings spaced three to five minutes apart. The PASDE recorders were started several minutes before the first firing of the sequence, at MET 2:19:22, as the crew proceeded through the test procedure checklist. Recording continued for 18:34.

Following the first PRCS test, a postdocking lighting calibration was conducted to fully account for the presence of Mir. The results of the this calibration were used to update camera iris settings for the second PRCS test, which began at 3:16:54. For the 
second test, the Kvant-II solar array was positioned at PASDE science team request to provide optimum photogrammetric viewing angles with respect to the six cameras. PASDE data recording was stopped after approximately 9:30 prior to completion of the jet firing sequences, when it was determined that the fields-of-view of four of the six PASDE cameras were most probably blocked. This test was repeated later in the mission to satisfy PASDE science objectives.

Prior to the beginning of the third PRCS test, an attitude reorientation of the docked Shuttle/Mir spacecraft using the PRCS jet system was flown. PASDE was used to record this test as a data collection event-of-opportunity and recorded 10:30 of data beginning at 5:00:35. The third PRCS test followed starting at MET 5:01:43. Data recording lasted 21:50, and downlink video from the PASDE cameras was available near the end of the test. The solar array was positioned as desired by PASDE, and the test occurred over Russian ground stations. Video downlink images of the Bay 6 root and tip cameras are shown in Figure 8 (compare with predicted Bay 6 views in Figure 7.

Orbit Sunlight Terminator and Solar Array Sun Track - Day-to-night and night-today terminator crossings were recorded to determine if thermal loading on the Kvant-II solar array could cause detectable motion. These data recordings were made on the fourth flight day between the second and third PRCS jet firing tests. For the day-tonight terminator, only the Bay 6 instrument was used, with the camera iris' set full open for the dark condition. Recording started at MET 4:21:28 and lasted for 2:00. Twentysix minutes later, at MET 4:21:54 the recorders on all three instruments were turned on for 5:14 to record the night-to-day terminator crossing.

The final PASDE data collection events on the STS-74 were of the Kvant-II solar array slews for sun tracking. Data was recorded twice, at MET 5:06:15 for 1:20, and at MET 5:06:24, for 8:35. Downlink of PASDE video was intermittently available during these data recordings, and structural response of the array to the slew maneuvers was visible. Structural response to sun track slews was also noted at various other times throughout the docked portions of the mission.

\section{Concluding Remarks}

The ISS Phase-I PASDE Risk Mitigation experiment was developed by the NASA Langley Research Center as a NASA Goddard Space Flight Center Hitchhiker payload. The PASDE flight hardware was designed, fabricated, assembled, tested, and delivered to Goddard for integration in eight months. The experiment hardware flew on the STS-74 Space Shuttle docking mission with the Russian Space Station Mir in November 1995. 113 minutes of Kvant-II solar array response video data was recorded during the mission. The results of the experiment will provide confidence to the ISS program that photogrammetric measurement methods can provide sufficient resolution and accuracy to meet appendage structural verification measurement requirements.

\section{Acknowledgments}

The PASDE flight experiment involved the dedicated efforts of many individuals and teams. In particular, the efforts of the Langley Project Manager Mr. John Fedors, the PASDE hardware design and development team, the PASDE mission operations team, the GSFC POCC operations team, and the Goddard GPP Mission Manager Mr. Gerry Daelemans are gratefully acknowledged.

\section{References}

[1] Pappa, R. S., "Eigensystem Realization Algorithm User's Guide for VAX/VMS Computers," NASA TM 109066, May 1994.

[2] "Hitchhiker Customer Accommodations and Requirements Specifications," NASA Goddard Spaceflight Center Document HHG730-1503-06, 1992.

[3] "Glo Experiment/Photogrammetric Appendage Structural Dynamics 
Experiment Payload, Payload

Integration Plan (PIP) Basic," NASA

Johnson Space Center Document

NSTS 21316, February 1995.

[4] Webster's Ninth New Collegiate

Dictionary, Merriam-Webster Inc.,

Springfield, MA., 1990.

[5] Slama, C. C., Theurer, C., and

Henriksen, S. W. Manual of

Photogrammetry, Fourth Edition,

Published by American Society of

Photogrammetry, Falls Church, VA., 1980.

[6] Brumfield,M. L., Pappa, R. S., Miller, J. B., and Adams, R. R., "Orbital Dynamics of the OAST-1

Solar Array Using Video

Measurements," Presented at the
AIAA/ASME/ASCE/AHS $26^{\text {th }}$

Structures, Structural Dynamics, and Materials Conference, Orlando, FL., April 15-17, 1985, AIAA Paper 850758-CP.

[7] Byrdsong, T. A., Adams, R. R., and Sandford, M. C., "Close-Range Photogrammetry Measurement of Static Deflections for an Aeroelastic Supercritical Wing," NASA TM4194, December 1990.

[8] Pappa, R. S., Gilbert, M. G., and Welch, S. S., "Simulation of the Photogrammetric Appendage Structural Dynamics Experiment," 14th International Modal Analysis Conference, Dearborn, MI., Feb. 12$15,1996$.

Table 1 - PASDE Hardware Design Requirements

\begin{tabular}{|c|c|}
\hline \multicolumn{1}{|c|}{ Item } & Requirement \\
\hline \hline Resolution & Measure 0.1" motions of the array tip \\
\hline \# of Array Measurements & Minimum of 6 needed to extract motion and determine modes shapes \\
\hline Data Recording & $1-2$ minutes prior/during excitation \\
& $3-5$ minutes following excitation \\
\hline Time Correlation & Video data time tagged to Shuttle events consistently for all events \\
\hline Sample Rate & $10 \mathrm{~Hz}$. or greater \\
\hline
\end{tabular}

Table 2 - STS 74 Mission Events

\begin{tabular}{|c||c||c|}
\hline $\begin{array}{c}\text { MET Ref. } \\
\text { (d:hh:mm) }\end{array}$ & $\begin{array}{c}\text { Record Time } \\
\text { (mm:ss) }\end{array}$ & \multicolumn{1}{|c|}{ Description } \\
\hline \hline $0: 00: 00$ & $00: 00$ & STS-74 launch at 7:30:43am EST, 11/12/95 \\
\hline $0: 03: 25$ & $00: 00$ & PASDE activation by Shuttle crew \\
\hline $1: 04: 45$ & $11: 35$ & Camera alignment verification and contingency data collection \\
\hline $2: 17: 52$ & $10: 47$ & Shuttle docking with Mir \\
\hline $2: 19: 22$ & $18: 34$ & PRCS Test \#1 \\
\hline $3: 16: 54$ & $09: 30$ & PRCS Test \#2 \\
\hline $4: 21: 28$ & $02: 00$ & Day-to-night terminator (Bay 6 canister only) \\
\hline $4: 21: 54$ & $05: 14$ & Night-to-day terminator \\
\hline $5: 00: 35$ & $10: 30$ & Shuttle/Mir attitude maneuver using Shuttle primary jets \\
\hline $5: 01: 43$ & $21: 50$ & PRCS Test \#3 \\
\hline $5: 06: 15$ & $01: 20$ & Solar array sun track slew maneuver \#1 \\
\hline $5: 06: 24$ & $08: 35$ & Solar array sun track slew maneuver \#2 \\
\hline- & $13: 31$ & Other data tape recording (recorder/commanding tests, etc.) \\
\hline \hline & $113: 26$ & Total on-orbit record time (115 minutes available) \\
\hline
\end{tabular}



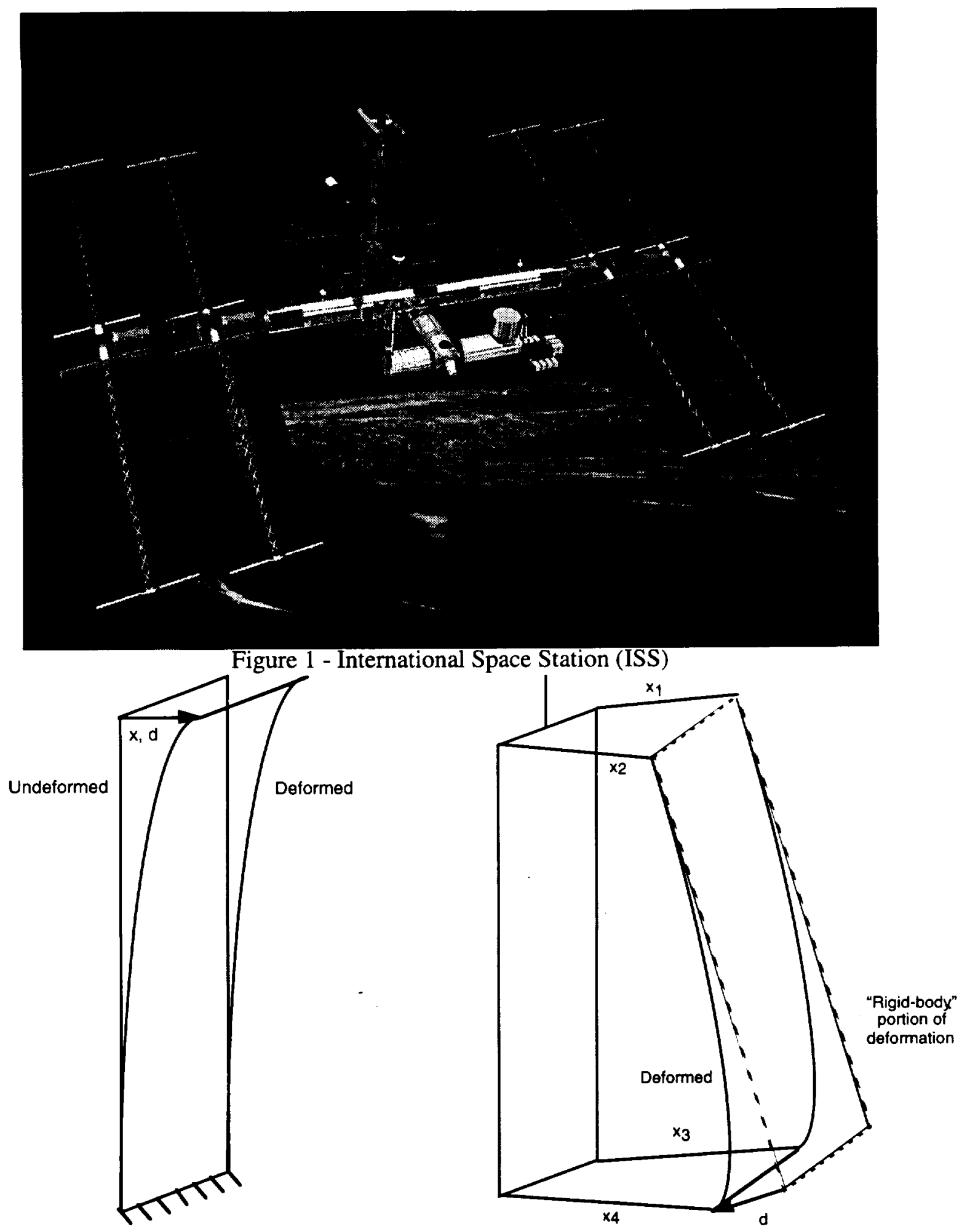

$x=$ photogrammetric measurement

$d=$ desired structural measurement

Figure 2 - Solar Array Flight Experiment (SAFE) structural measurement.

$x=$ photogrammetric measurement

$d=$ desired structural measurement

Figure 3 - Relative motion case structural measurement. 


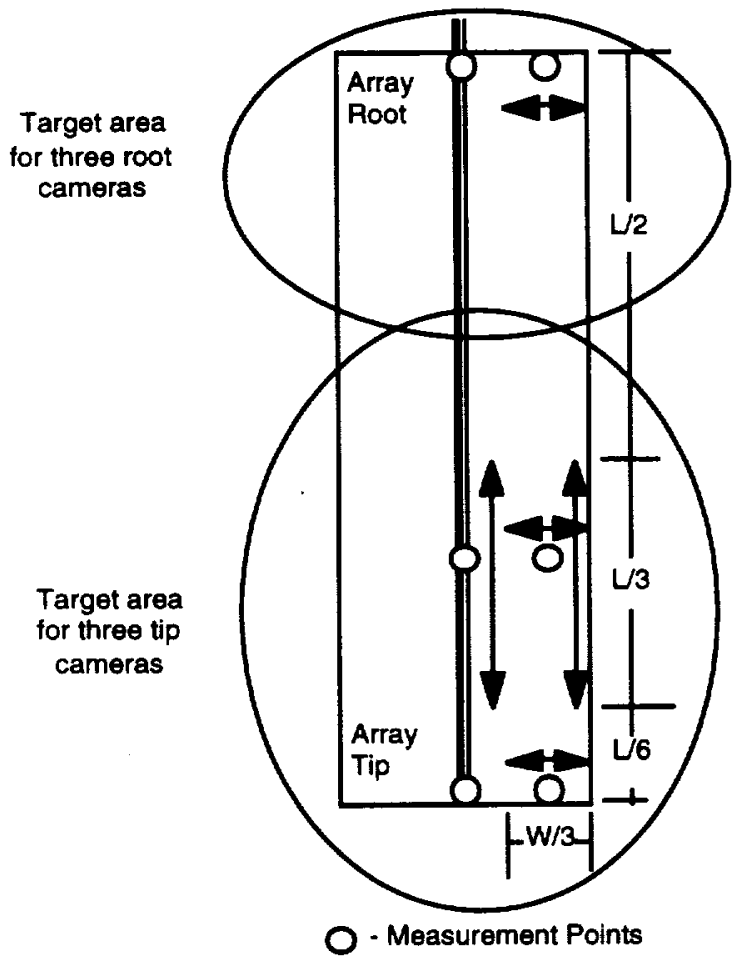

Figure 4 - Definition of minimum number and location of measurement points.

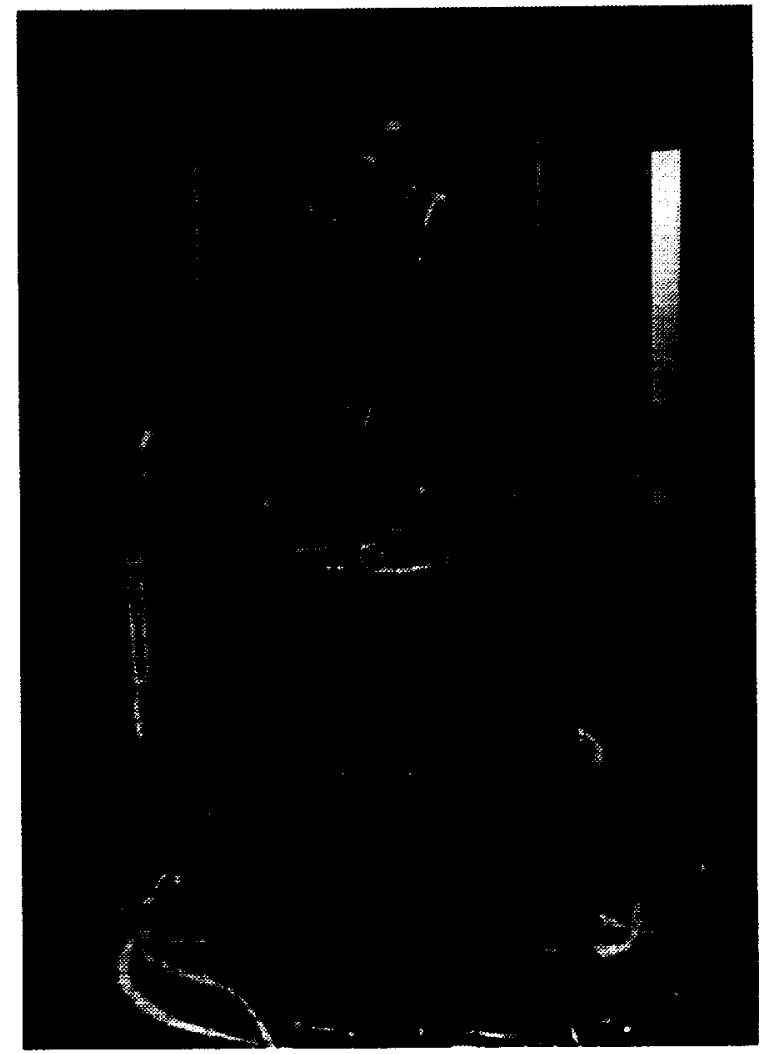

Figure 5 - PASDE flight hardware prior to Hitchhiker canister integration.

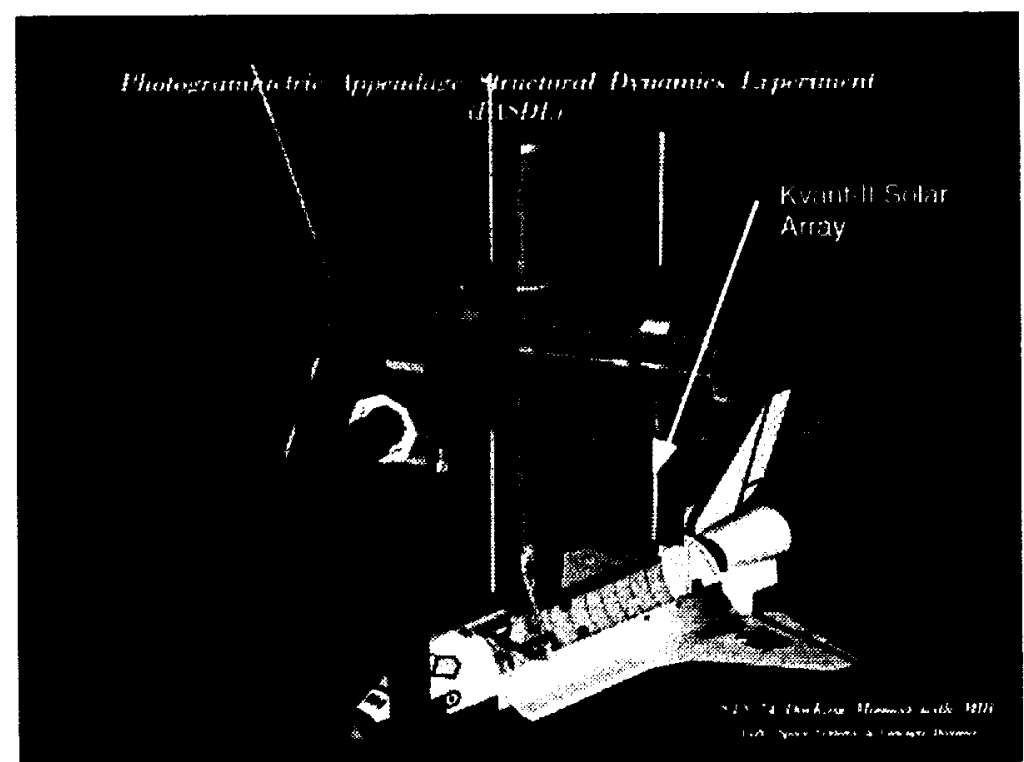

Figure 6 - STS-74 Shuttle/Mir configuration. 
(a)

Figure 7 - Expected Bay 6 views of Kvant-II solar array: (a) Tip Camera, (b) Root Camera

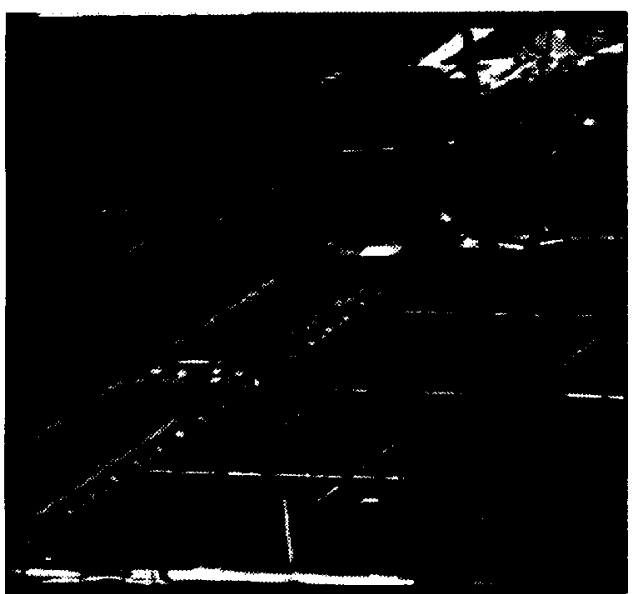

(a)

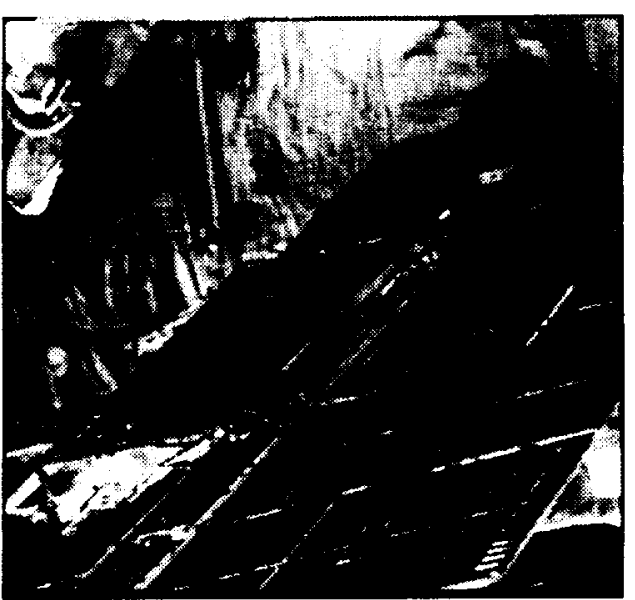

(b)

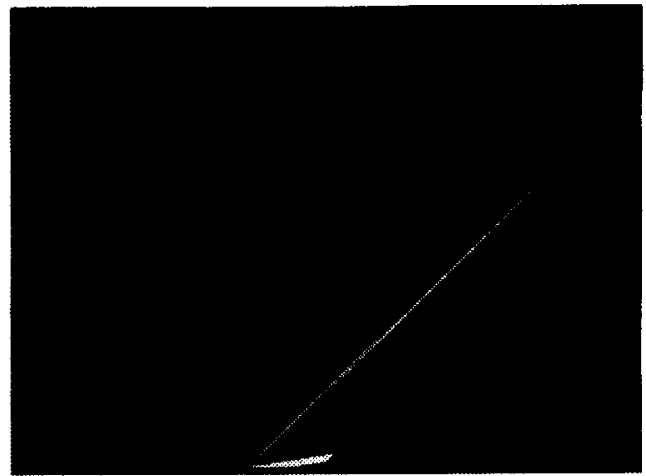

(b)

Figure 8 - Bay 6 downlink video images: (a) Tip Camera, (b) Root Camera 


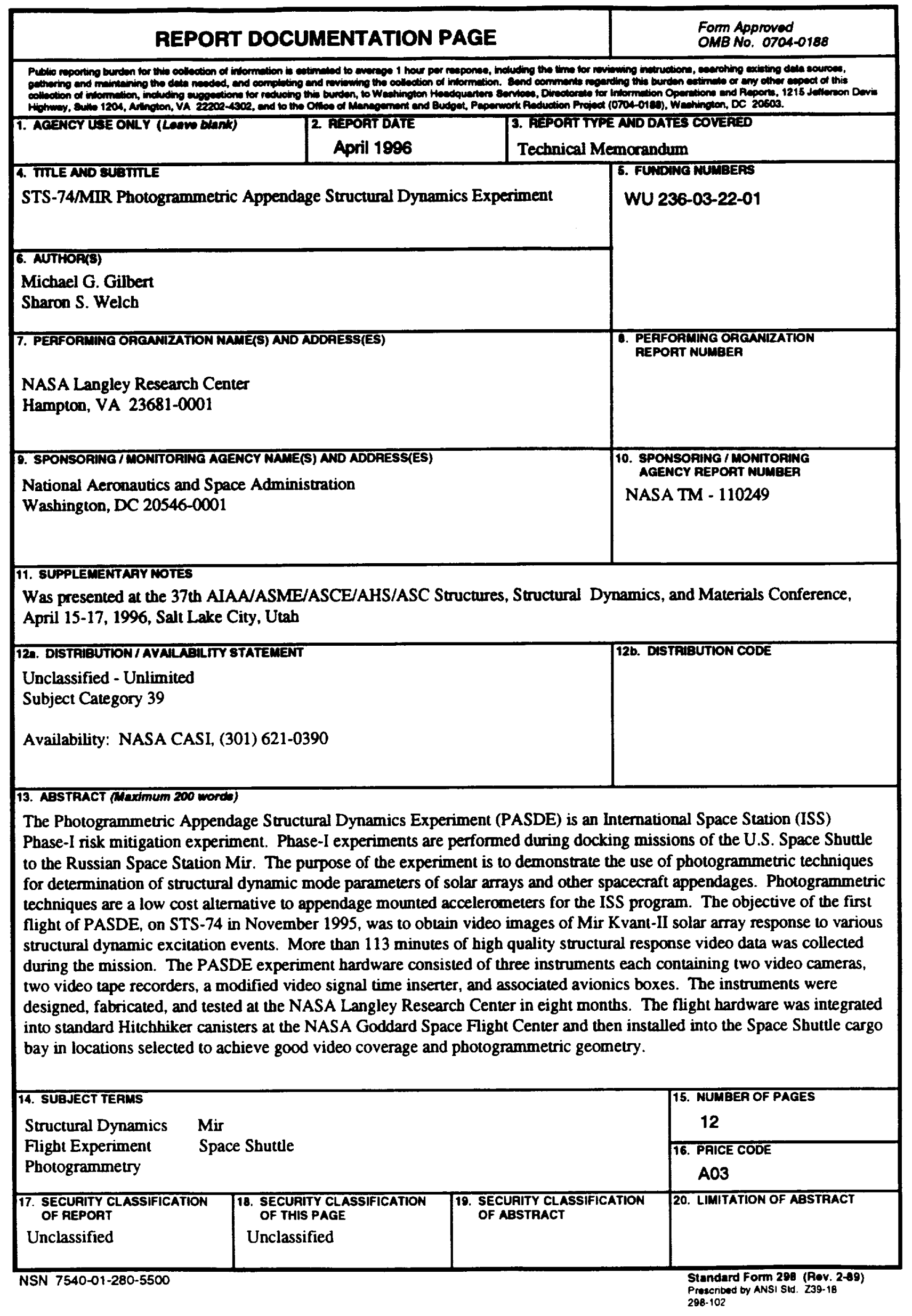





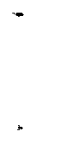

